\title{
Humoral Response among Patients with Interstitial Lung Disease Vaccinated with the BNT162b2 SARS- Cov-2 vaccine - A Prospective Cohort Study
}

Barak Pertzov ( $\sim$ pertzovb@gmail.com)

Rabin Medical Center

Einat Shmueli

Schneider Children's Medical Center of Israel

Haim Ben Zvi

Rabin Medical Center

Amir Massarweh

Rabin Medical Center

Tamar Barkan

Rabin Medical Center

Assaf Ness

Rabin Medical Center

Yael Shostak

Rabin Medical Center

Lev Freidkin

Rabin Medical Center

Osnat Shtraichman

Rabin Medical Center

Mordechai R Kramer

Rabin Medical Center

\section{Research Article}

Keywords: Humoral Response, Interstitial Lung Disease, BNT162b2, SARS-Cov-2 vaccine

Posted Date: December 29th, 2021

DOI: https://doi.org/10.21203/rs.3.rs-1201596/v1

License: (c) (1) This work is licensed under a Creative Commons Attribution 4.0 International License.

Read Full License 


\section{Abstract}

Background: Patients with interstitial lung disease (ILD) are at high risk of severe COVID-19 infection. Additionally, their anti-inflammatory and antifibrotic treatment may cause immunosuppression. Nevertheless, their ability to mount an adequate immune response to messenger RNA SARS-CoV-2 vaccines was not evaluated. We aimed to evaluate the humoral response after the BNT162b2 vaccine among idiopathic pulmonary fibrosis (IPF) patients treated with antifibrotic therapy and among non-IPF ILD patients treated with anti-inflammatory therapy.

Methods: We conducted an observational prospective cohort study to evaluate the rate of anti-spike (S$\mathrm{IgG}$ ) antibodies after two doses of the BNT162b2 vaccine in patients with ILD. The cohort included 40 patients with idiopathic pulmonary fibrosis (IPF) treated with anti-fibrotic therapy and 29 patients with non-IPF ILD treated with anti-inflammatory therapy. For S-IgG titer measurement one serology test was drawn from all patients 4-6 months after the second vaccine dose. Two age and sex matched control groups were created from a healthy control cohort of 107 patients. The study was conducted in Rabin Medical Center (Israel) between June to August 2021.

Results: All patients in the anti-fibrotic arm were seropositive (40/40), corresponding to the matched control group ( $P=1.0)$. The antifibrotic arm had a significantly lower median antibody titer in comparison to the matched control group (361.10 [ IQR, 207-811] AU/ml vs 820.75 [IQR, 459-1313] AU/ml; P<0.001). Only $48.3 \%(14 / 29)$ of patients in the anti-inflammatory arm were seropositive in comparison to $100 \%$ $(29 / 29)$ in the healthy control group $(P<0.001)$. The anti-inflammatory arm had a significantly lower median antibody titer in comparison to the healthy control group (39.6 [ IQR, 4.25-165] AU/ml vs 970.1 [IQR, 505-1926] AU/ml; P<0.001).

Conclusion: IPF patients treated with antifibrotic therapy mount an adequate immune response after 2 doses of the BNT162b2 vaccine, maintain a $100 \%$ seropositivity rate, 4-6 months after vaccination. However, their antibody titer was reduced in comparison to a healthy control group. Among patients with non-IPF ILD, treated with anti-inflammatory therapy, $48 \%$ were seronegative 4-6 months after the second vaccine dose, moreover treatment with rituximab caused significant immunosuppression, even in comparison to other anti-inflammatory treatments.

\section{Introduction}

Coronavirus disease 2019 (COVID-19), caused by severe acute respiratory syndrome coronavirus 2 (SARS-Cov-2), is an ongoing global pandemic (1). Several vaccines are currently used for disease prevention, the most common are the mRNA vaccines. In contrast to healthy volunteers who mount an adequate immune response after vaccination, patients with chronic disease, who are treated with antiinflammatory therapy have a reduced immune response (2-5). Patients with interstitial lung disease (ILD) are treated with both anti-inflammatory and antifibrotic therapy in accordance with their underlying disease. Idiopathic pulmonary fibrosis (IPF), the most common of the idiopathic interstitial pneumonias, 
is a fibrotic disease treated solely with antifibrotic therapy. Two antifibrotic drugs are currently approved for IPF therapy, nintedanib, which is a tyrosine kinase inhibitor that exerts an inhibitory effect on fibroblast growth factor receptor (FGFR), endothelial growth factor receptor and platelet-derived growth factor receptor (PDGFR) $(6,7)$, and pirfenidone, which is an antifibrotic drug that reduces fibrosis in lung, hepatic, kidney, and cardiac tissue. Pirfenidone works partially by inhibition of transforming growth factor beta (TGF- $\beta$ ), however, its direct molecular target is unknown $(8,9)$. The impact of antifibrotic treatment on the humoral response after mRNA vaccine have yet to be evaluated in clinical trials. In this trial we aim to evaluate the humoral response after the BNT162b2 vaccine among IPF patients treated with antifibrotic therapy and among non-IPF ILD patients treated with anti-inflammatory therapy.

\section{Methods}

We conducted an observational prospective cohort study at Rabin Medical Center between June to August 2021, to evaluate the humoral response to the BNT162b2 vaccine among patients with interstitial lung disease. Patients eligible for inclusion were adults ( $\geq 18$ years) who were vaccinated with two doses of the BNT162b2 vaccine, diagnosed with idiopathic pulmonary fibrosis treated with anti-fibrotic therapy (nintedanib or pirfenidone) or non-IPF ILD patients treated with anti-inflammatory treatment (e.g., glucocorticoids, antimetabolites, and rituximab). Exclusion criteria were patients who were previously infected with COVID-19 (as documented by positive PCR nasal swab). All patients signed an informed consent form and the study was approved by the institutional review board at Rabin Medical Center (RMC-0294-21).

\section{Data collection}

Patients were invited to participated in the study when they arrived for their routine evaluation in the ILD clinic or by a public invitation to participate that was sent to all ILD patients in the country, with aid of the Israeli ILD patient foundation. Patients that agreed to participate and signed an informed consent form were invited to the pulmonary institute for serology testing. From each patient one serology test tube was drawn and sent to the lab for analysis. Relevant demographic and clinical data, including immunosuppressive drug regimens and vaccination dates were recorded from the electronic medical records at Rabin Medical Center (RMC).

\section{Treatment arms}

This observational study included two treatment arms and one control arm: an antifibrotic treatment arm included IPF patients and an anti-inflammatory treatment arm included non-IPF ILD patients The control arm included health care workers from RMC and a cohort of healthy volunteers that were recruited as a control group for a similar study at RMC (10) and were also used as a control group in this study. For 
each evaluated arm a 1:1 age and gender matched control group was created from the healthy control cohort.

\section{Blood sample processing}

Whole blood samples were drawn from participants at study visit. Serum was separated by centrifugation, aliquoted and stored at $-20^{\circ} \mathrm{C}$ until the serological assay was performed.

SARS-CoV-2 IgG II Quant assay (Abbott Ireland Diagnostic Division) was performed using the ARCHITECT ${ }^{\circledR}$ i2000SR immunoassay analyzer in accordance with the manufacturer's package insert. The assay is a chemiluminescent microparticle immunoassay (CMIA) used for the quantitative determination of immunoglobulin class $\mathrm{G}(\mathrm{IgG})$ antibodies to the receptor binding domain (RBD) of S1 subunit of the spike protein of SARS-CoV-2 (S-IgG) in human serum and plasma samples. In the assay, SIgG antibodies bind to antigen coated paramagnetic microparticles, and anti-human IgG acridiniumlabeled conjugate is added to create a reaction mixture. Following further processing, the resulting chemiluminescent reaction is measured as a relative light unit (RLU), with the detected RLU directly related to the amount of S-IgG in the sample. S-IgG titers of $50 \mathrm{AU} / \mathrm{mL}$ and greater in the immunoassay test are interpreted as positive (11-13).

\section{Outcomes}

The primary outcome was to assess the rate of seropositivity in ILD patients following the BNT162b2 vaccine, as measured by S-IgG antibodies present 4-6 months after receiving two vaccine doses. Positive S-IgG antibody titer was defined as $\geq 50 \mathrm{AU} / \mathrm{ml}$. Secondary outcomes were the median titer and geometric mean titer (GMT) of S-IgG antibodies and identification of independent predictive factors for negative serologic response by multivariate analysis.

\section{Statistical analysis}

Demographic and clinical baseline characteristics were compared with the Student's t-test, chi-squared test and Mann-Whitney $U$ test, as appropriate. The primary outcome was analyzed with the chi-squared test. Antibody level was presented as median (IQR) and GMT and was analyzed with the Mann-Whitney U test and Student's t-test, respectively. Comparison between the antibody titer of different treatment groups (e.g control, antifibrotic, antimetabolites+glucocorticoid and rituximab) was analysed by LOG10 conversion followed by one way ANOVA with Bonferroni correction for multiple comparisons.

Multivariate analysis for independent predictors of negative serologic response was conducted with linear regression. SPSS version 27 (IBM corp., Armonk, NY) was used for statistical analysis.

\section{Results}


Overall, 176 participants were included in the final analysis. 40 patients in the anti-fibrotic arm, 29 patients in the anti-inflammatory arm and 107 patients in the control arm. Patients with IPF that were treated with anti-fibrotic therapy (anti-fibrotic arm) had a median (IQR) age of 71 (67-75) and 27 patients $(67.5 \%)$ were men. The matched control group had a median (IQR) age of $69(63-72)$ and $16(40 \%)$ were men. All patients in the anti-fibrotic arm were seropositive (40/40), corresponding to the matched control group $(P=1.0)$. However, the antifibrotic arm had a significantly lower median antibody titer in comparison to the matched control group (361.10 [ IQR, 207-811] AU/ml vs 820.75 [IQR, 459-1313] AU/ml; $P<0.001)$. The median (IQR) time between the second vaccine dose and the serology test was 173 days (168-182) in the anti-fibrotic arm and 128.55 days $(123-142)$ in the controls $(P<0.001)$ (Table 1$)$

Patients with non-IPF ILD treated with anti-inflammatory treatment had a median (IQR) age of 67 (55-73) and 11 (38\%) were men. The matched control group had a median (IQR) age of 70 (56-72) and 11 (38\%) were men. Within the anti-inflammatory arm, all patients (29/29) were treated with antimetabolites and/or oral corticosteroids, 8 patients were also treated with rituximab. Only $48.3 \%(14 / 29)$ of patients in the anti-inflammatory arm were seropositive in comparison to $100 \%(29 / 29)$ in the healthy control group $(P<0.001)$. The anti-inflammatory arm had significantly lower median antibody titer in comparison to the matched control group (39.6 [ IQR, 4.25-165] AU/ml vs 970.1 [IQR, 505-1926] AU/ml; $P<0.001)$. The median (IQR) time between the second vaccine dose and the serology test was 179 days (168-184) in the anti-inflammatory arm and 137 days $(122-158)$ in the controls $(P<0.001)$ (Table 2$)$. When compared to the anti-inflammatory arm, the antifibrotic arm had a significantly higher rate of seropositivity and antibody titer (supplementary, Table S1).

Overall patients in the control group had the highest median antibody titer (1102, IQR 611-2034) followed by patients treated with antifibrotic drugs (361, IQR 207-811), antimetabolites and corticosteroids (61.8, IQR 21-214) and rituximab (3.5, IQR 0-4.3), overall difference and pairwise comparison were all significant $(P<0.001$ in all between-group comparisons) (Figure 1). Multivariate analysis showed that antiinflammatory treatment significantly reduced the immune response while, age, antifibrotic therapy and the number of days post vaccination did not have a significant effect (Table 3 ).

Table 1

Baseline characteristics and antibody titers among patients treated with anti-fibrotic treatment and matched control 


\begin{tabular}{|llll|}
\hline & $\begin{array}{l}\text { Anti-fibrotic Treatment } \\
(\mathbf{n = 4 0})\end{array}$ & $\begin{array}{l}\text { Matched control } \\
(\mathbf{n = 4 0})\end{array}$ & P value \\
\hline Age, median (IQR), y & $71(67-75)$ & $69(63-72)$ & 0.02 \\
\hline Male sex (\%) & $27(67.5)$ & $16(40)$ & 0.01 \\
\hline IgG titer, median (IQR), Au/ml & $361.10(207-811)$ & $820.75(459-1313)$ & 0.001 \\
\hline IgG titer GMT (SD) & $441.26 \pm 3.15$ & $835.41 \pm 2.07$ & 0.004 \\
\hline Seropositive (\%) & $40(100)$ & $40(100)$ & 1.0 \\
\hline Days post vaccination, median (IQR) & $173(168-182)$ & $128.55(123-142)$ & $<0.001$ \\
\hline
\end{tabular}

Table 2

Baseline characteristics and antibody titers among patients treated with anti-inflammatory treatment and matched control

\begin{tabular}{|lllc|}
\hline & $\begin{array}{l}\text { Anti-inflammatory Treatment } \\
(\mathbf{n = 2 9})\end{array}$ & $\begin{array}{l}\text { Matched Control } \\
(\mathbf{n = 2 9})\end{array}$ & P value \\
\hline Age, median (IQR), $\mathbf{y}$ & $67(55-73)$ & $70(56-72)$ & 0.86 \\
\hline Male sex (\%) & $11(38)$ & $11(38)$ & 1.0 \\
\hline Anti-inflammatory treatment & & & \\
\hline Antimetabolites and OCS & 21 & NA & \\
\hline rituximab & 8 & $970.10(505-1926)$ & $<0.001$ \\
\hline IgG titer, median (IQR), Au/ml & $39.60(4.25-165)$ & $1006.93 \pm 2.45$ & $<0.001$ \\
\hline IgG titer GMT (SD) & $23.70 \pm 12.73$ & $29(100)$ & $<0.001$ \\
\hline Seropositive (\%) & $14(48.3)$ & $137(122-158)$ & $<0.001$ \\
\hline Days post vaccination, median (IQR) & $179(168-184)$ & & \\
\hline OCS: Oral glucocorticoid & & & \\
\hline
\end{tabular}

Table 3

Multivariate analysis: Predictors for reduced humeral response 


\begin{tabular}{|llll|}
\hline & OR & Cl & P value \\
\hline Age & -13.77 & $-31.62-4.08$ & 0.13 \\
\hline Anti-fibrotic Tx & -311.76 & $-1002.82-379.29$ & 0.37 \\
\hline Anti-inflammatory Tx & -1287.21 & $-1992.15-(-582.81)$ & $<0.001$ \\
\hline Time from vaccine & -1.01 & $-10.51-8.48$ & 0.83 \\
\hline
\end{tabular}

\section{Discussion}

This study evaluated the humoral response among IPF patients treated with antifibrotic therapy and nonIPF ILD patients treated with anti-inflammatory treatment in comparison to an age and sex matched healthy control group, after 2 doses of the BNT162b2 SARS-CoV-2 vaccine. The study showed that IPF patients treated with antifibrotic therapy mount an adequate immune response to the BNT162b2 SARSCoV-2 vaccine. However, with a reduced antibody titer in comparison to the control group. To our knowledge this is the first study to show that antifibrotic treatment enables a suitable immune response to the COVID-19 mRNA vaccine. The antibody titer in both treatment arms was evaluated 4-6 months after the second vaccine dose, at this time point the level of S-IgG antibodies is reduced in comparison to the early post vaccination period, since the antibody titer slowly declines after receiving the second vaccine dose (14). Nevertheless, all patients in the anti-fibrotic group were seropositive at this time point, which further strengthens the conclusion that these patients mount and maintain an adequate immune response 4-6 months after vaccination A recently published preprint that evaluated the impact of chronic disease on the humoral response after mRNA vaccine, found that ILD was an independent risk factor for reduced immunity independent of the patients medication use (5). Furthermore, several trials have shown that in addition to their anti-fibrotic effect both pirfenidone and nintedanib have an anti-inflammatory effect, which can interfere with humoral response. In pirfenidone by inhibition of dendritic cells, macrophages, neutrophils, eosinophils and T lymphocytes (15) and in nintedanib by blocking $\mathrm{T}$ cell activation $(6,16)$. Although, we cannot ascertain whether the mildly reduced antibody titer in treated IPF patients was the result of the chronic interstitial lung disease or the antifibrotic treatment, it was most probably the result of both factors. Due to the differences in drug mechanism of action among the antifibrotic drugs, we also compared the titer of S-IgG antibodies in pirfenidone and nintedanib users and found no differences (Figure 2). Finally, we evaluated the effect of different treatment regimens on the humoral response and showed that anti-inflammatory treatment with antimetabolites, glucocorticoids and rituximab causes a far greater reduction in immunity, in comparison to antifibrotic treatment. This further emphasizes the difference in mechanism of action of the antifibrotic drugs, which lead to improvement in clinical outcomes in IPF patients, in contrast to anti-inflammatory treatment (17).

The anti-inflammatory arm showed a significantly reduced humoral response in comparison to the control group. These results are in agreement with several studies that showed reduced humoral response in patients with inflammatory bowel disease, connective tissue disease and solid organ recipients, who were treated with anti-inflammatory therapy (2-4). Patients treated with rituximab 
showed significant immunosuppression, even in comparison to other anti-inflammatory treatments. Comparable results were seen in similar trials, this profound immunosuppression should be taken under consideration when this drug is prescribed in the COVID-19 era (18-20).

This study has several limitations, there is a one-month difference in the time between the second vaccine dose and the serology test between the control group and the anti-fibrotic and anti-inflammatory groups. Furthermore, between the anti-fibrotic arm and the matched control group was a small yet statistically significant age difference. However, we were able to show in the multivariate analysis that age and the number of days from the second vaccine did not influence the antibody titer (Table 3 ). The sample size was small, 40 patients in the antifibrotic arm and 29 patients in the anti-inflammatory arm. Nevertheless, this study was the first to describe IPF patients specifically and for this relatively rare disease the sample size allows for statistical analysis and meaningful conclusions. Finally, the study evaluated anti spike $\lg \mathrm{G}$ antibodies and not neutralizing antibodies.

In conclusion, IPF patients treated with antifibrotic treatment mount an adequate immune response after 2 doses of the BNT162b2 vaccine and maintain 100\% seropositivity rate 4-6 months after vaccination. However, the antibody titer was reduced in comparison to an age matched healthy control group. Among patients with non-IPF ILD, treated with anti-inflammatory therapy, 48\% were seronegative 4-6 months after the second vaccine dose, moreover treatment with rituximab caused significant immunosuppression even in comparison to other anti-inflammatory treatments.

\section{Declarations}

\section{Ethics approval and consent to participate:}

All patients signed an informed consent form, and the study was approved by the institutional review board at Rabin Medical Center (RMC-0294-21).

\section{Consent for publication:}

Not applicable. Availability of data and materials: The datasets generated and/or analysed during the current study are available from the corresponding author on reasonable request.

\section{Competing interests:}

The authors declare that they have no competing interests.

\section{Funding:}

None 


\section{Authors' contributions:}

$B P, E S, O S$ and MRK conceived and planned the study, AM, TB, AN, YS and LF recruited the patients and preformed the serology testing, HBZ completed the microbiology work. BP, ES, OS, MRK and YS wrote and revised the manuscript.

\section{Acknowledgements:}

We would like to thank Ms. Vidi Bar-Nathan, the IPF patient foundation and the ILD patients in Israel for their cooperation. Without their contribution and effort this study would not have been completed. The authors express their gratitude to Ms. Dalia Dawn Orkin for her English language contributions and editing services.

\section{References}

1. CDC. Coronavirus Disease 2019 (COVID-19) [Internet]. Centers for Disease Control and Prevention. 2020 [cited 2021 Feb 20]. Available from: https://www.cdc.gov/coronavirus/2019-ncov/index.html

2. Shostak Y, Shafran N, Heching M, Rosengarten D, Shtraichman O, Shitenberg D, et al. Early humoral response among lung transplant recipients vaccinated with BNT162b2 vaccine. Lancet Respir Med. 2021 Jun;9(6):e52-3.

3. Tang W, Gartshteyn Y, Ricker E, Inzerillo S, Murray S, Khalili L, et al. The Use of COVID-19 Vaccines in Patients with SLE. Curr Rheumatol Rep. 2021 Nov 12;23(11):79.

4. Melmed GY, Botwin GJ, Sobhani K, Li D, Prostko J, Figueiredo J, et al. Antibody Responses After SARS-CoV-2 mRNA Vaccination in Adults With Inflammatory Bowel Disease. Ann Intern Med. 2021 Oct 12;

5. Liao S-Y, Gerber AN, Zelarney P, Make B, Wechsler ME. Impaired SARS-CoV-2 mRNA vaccine antibody response in chronic medical conditions: a real-world data analysis. medRxiv. 2021 Jan $1 ; 2021.08 .03 .21261483$.

6. Wollin L, Wex E, Pautsch A, Schnapp G, Hostettler KE, Stowasser S, et al. Mode of action of nintedanib in the treatment of idiopathic pulmonary fibrosis. Eur Respir J. 2015 May;45(5):1434-45.

7. Epstein Shochet G, Bardenstein-Wald B, McElroy M, Kukuy A, Surber M, Edelstein E, et al. Hypoxia Inducible Factor 1A Supports a Pro-Fibrotic Phenotype Loop in Idiopathic Pulmonary Fibrosis. Int J Mol Sci. 2021 Mar 24;22(7):3331.

8. Knüppel L, Ishikawa Y, Aichler M, Heinzelmann K, Hatz R, Behr J, et al. A Novel Antifibrotic Mechanism of Nintedanib and Pirfenidone. Inhibition of Collagen Fibril Assembly. Am J Respir Cell Mol Biol. 2017 Jul 1;57(1):77-90. 
9. Schaefer CJ, Ruhrmund DW, Pan L, Seiwert SD, Kossen K. Antifibrotic activities of pirfenidone in animal models. Eur Respir Rev. 2011 Jun;20(120):85-97.

10. Eliakim-Raz N, Massarweh A, Stemmer A, Stemmer SM. Durability of Response to SARS-CoV-2 BNT162b2 Vaccination in Patients on Active Anticancer Treatment. JAMA Oncol. 2021 Nov 1;7(11):1716-8.

11. Abbott ARCHITECT SARS-CoV-2 IgG II Quant Reagent Instructions for Use. 2020 Dec;

12. Meschi S, Colavita F, Bordi L, Matusali G, Lapa D, Amendola A, et al. Performance evaluation of Abbott ARCHITECT SARS-CoV-2 IgG immunoassay in comparison with indirect immunofluorescence and virus microneutralization test. J Clin Virol. 2020 Aug;129:104539.

13. administrator J website. Serology tests for COVID-19 [Internet]. COVID-19 Testing Toolkit. [cited 2021 Dec 12]. Available from: https://www.centerforhealthsecurity.org/covid19TestingToolkit/serology/Serology-based-tests-for-COVID-19.html

14. Levin EG, Lustig Y, Cohen C, Fluss R, Indenbaum V, Amit S, et al. Waning Immune Humoral Response to BNT162b2 Covid-19 Vaccine over 6 Months. N Engl J Med. 2021 Dec 9;385(24):e84.

15. Ruwanpura SM, Thomas BJ, Bardin PG. Pirfenidone: Molecular Mechanisms and Potential Clinical Applications in Lung Disease. Am J Respir Cell Mol Biol. 2020 Apr 1;62(4):413-22.

16. Ubieta K, Thomas MJ, Wollin L. $<\mathrm{p}>$ The Effect of Nintedanib on T-Cell Activation, Subsets and Functions</p>. DDDT. 2021 Mar 8;15:997-1011.

17. Prednisone, Azathioprine, and N-Acetylcysteine for Pulmonary Fibrosis. New England Journal of Medicine. 2012 May 24;366(21):1968-77.

18. Kroon FPB, Najm A, Alunno A, Schoones JW, Landewé RBM, Machado PM, et al. Risk and prognosis of SARS-CoV-2 infection and vaccination against SARS-CoV-2 in rheumatic and musculoskeletal diseases: a systematic literature review to inform EULAR recommendations. Ann Rheum Dis. 2021 Dec 7; annrheumdis-2021-221575.

19. Avouac J, Miceli-Richard C, Combier A, Steelandt A, Fogel O, Mariaggi AA, et al. Risk factors of impaired humoral response to COVID-19 vaccination in rituximab treated patients. Rheumatology (Oxford). 2021 Nov 2;keab815.

20. Felten R, Gallais F, Schleiss C, Chatelus E, Javier R-M, Pijnenburg L, et al. Cellular and humoral immunity after the third dose of SARS-CoV-2 vaccine in patients treated with rituximab. Lancet Rheumatol. 2021 Nov 8;

\section{Figures}

\section{Figure 1}

Antibody titer according to treatment type 


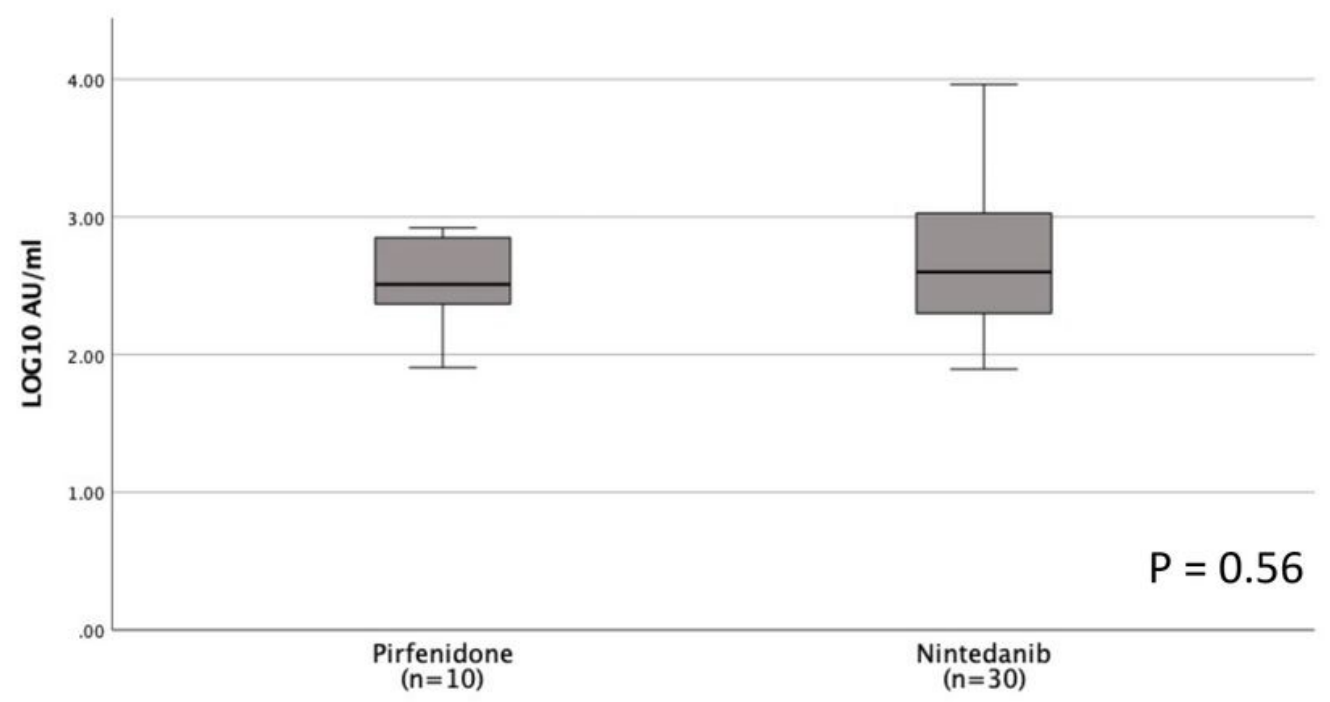

Figure 2

Comparison of S-IgG antibody titer in between patients treated with nintedanib vs pirfenidone

\section{Supplementary Files}

This is a list of supplementary files associated with this preprint. Click to download.

- supplementaryILDserology.docx 